\title{
Peak Stress Relief of Cross Folding Origami
}

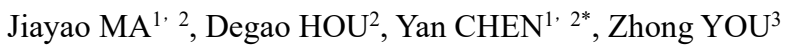 \\ ${ }^{1}$ Key Laboratory of Mechanism and Equipment Design of Ministry of Education, Tianjin University, Tianjin 300072, \\ China \\ ${ }^{2}$ School of Mechanical Engineering, Tianjin University, Tianjin 300072, China \\ ${ }^{3}$ Department of Engineering Science, University of Oxford, Parks Road, Oxford, OX1 3PJ, UK
}

\begin{abstract}
In this paper, cross folding of an elastic sheet, a common folding method in packaging a deployable antenna reflective membrane, was studied numerically with the aim of eliminating material failure in the sheet. The deformation, stress distribution, and peak stress of the sheet, as well as the effects of geometrical parameters on those properties were systematically investigated. Then two methods, i.e., central hole method and central slit method, were explored to reduce the peak stress caused by cross folding. A central slit parallel to the first folding crease was found to be most effective in reducing the peak stress.
\end{abstract}

Key words: deployable antenna reflective membrane; cross folding; stress concentration; peak stress relief.

\section{Introduction}

Deployable antenna reflectors are a typical kind of deployable structures in aerospace engineering, widely used for telecommunication, earth observation and other missions [1-6]. An antenna reflector is usually composed of a reflective membrane and a foldable ring that supports the membrane. Membrane has gained increasing popularity for two reasons: it can be easily packaged and its surface accuracy can be achieved by suitable tension upon deployment [7-11]. An antenna

\footnotetext{
${ }^{*}$ Corresponding author, email: yan_chen@tju.edu.cn, phone number: +86 2285356291.
} 
reflector with a diameter greater than 4 meters would have to be folded into smaller dimensions in order to be fitted into a launch vehicle and subsequently deployed once it reaches its orbit [7]. Various approaches have been adopted to fold an antenna reflector. Taking the CRTS reflector [12] as an instance, the ribs are rolled inward and packed in a zig-zag way as a folded umbrella, whereas the membrane is folded in between the ribs. While this folding scheme is able to achieve a large folding ratio, it unavoidably involves two folding creases being crossed each other in the membrane, leading to the formation of a vertex in the crossed area. This phenomenon is not limited in folding the antenna reflector membranes, but a general issue when four or more folding creases are met at a vertex in origami engineering structures with initially flat or curved surfaces [13-15].

For a membrane surface made of elastic materials, such as silicone rubber based materials which are recently being explored for their capability of being deployed into a desirable shape without cable tensioning, cross folding will cause localized energy and stress/strain concentrations at folding ridges and point-like vertices [16]. The concentrations at such singularities could cause irrecoverable plastic deformations, which in turn affect the accuracy of the deployed surfaces. Hence an in-depth understanding of the formation of vertices and ridges, their mechanical properties and influencing factors are very important for implementation of such new materials. Although bending of an elastic sheet is a thoroughly studied problem in elasticity, there is little literature related to cross folding. Therefore, the objective of this paper is to numerically model the cross folding of an flat elastic sheet in order to study the deformation, stress distribution, and peak stress of the sheet and how the geometrical parameters of the sheet influence those properties, and hence to give indications about crease designs which can be used to avoid such stress concentrations.

The layout of the paper is as follows. The geometry of the elastic sheet and the finite element 
modelling procedure are first presented in Section 2. Section 3 gives the deformation and stress analyses of a cross-folded sheet. Subsequently two peak-stress relief methods are proposed and analyzed in Section 4. Finally is the conclusion in Section 5 which ends the paper.

\section{Geometry and Finite Element Modelling}

A square sheet of side length $100 \mathrm{~mm}$ was considered in this study. After several rounds of trial-and-error, the following procedure was found to naturally imitate a folding motion and to effectively cross fold a sheet. As illustrated in Fig. 1, a sheet was first folded vertically along folding path BB' (Step 1) and then horizontally along AO (Step 2), resulting in a cross formed at the center $(\mathrm{O})$.

The two-step folding procedure of a sheet was controlled by 12 circular rigid bodies attached to it as shown in Fig. 1. Each rigid body was $5 \mathrm{~mm}$ in diameter and had a reference point assigned to it to control its movement. To achieve a natural folding motion, all reference points were placed at the mid surface of the sheet. Several simulations were conducted to determine the number and placement of the rigid bodies. It was found that the rigid bodies at the sides (1-6) were not enough to fold up the sheet, and hence two more columns of inner rigid bodies (7-12) with a position of $d$ from BB' were added.

The folding motion was enabled by assigning boundary conditions to the rigid bodies [17]. Specifically, in step 1, the rigid bodies on the left-hand side and those on the right-hand side rotated about the Y-axis by $90^{\circ}$ towards the center, but in opposite directions so that the sheet could be folded up. Accordingly, all the reference points associated with the rigid bodies were completely fixed in space except for the rotational degree of freedom about the Y-axis. And a positive value of $90^{\circ}$ was assigned to the rigid bodies on the left-hand side, whereas a negative value of identical 
magnitude to those on the right-hand side. In step 2, the rigid bodies along AA' $(2,5,8$, and 11$)$ were fixed with no translation or rotation. And the upper rigid bodies $(1,4,7,10)$ and the lower ones $(3,6,9,12)$ rotated about the Z-axis towards the center by $90^{\circ}$. This was also achieved by a similar setup as step 1 . Notice that the expected folding mode was achieved by a proper placement of rigid bodies, without the introduction of geometric imperfection or perturbation in the sheet.

The sheet thickness $t$ and position of the inner rigid bodies $d$ were respectively varied between $0.1 \mathrm{~mm}$ and $1 \mathrm{~mm}$ and between $15 \mathrm{~mm}$ and $37.5 \mathrm{~mm}$ to investigate their effects on the deformation and stress of the sheet. Note that the focus of the study was how to keep the sheet in the elastic range, without considering what happened when the material went into plastic. Moreover, the peak stress in the sheets studied here was mostly within 2-3 MPa, and in this range the difference between Mises and Tresca stress was found to be small. Therefore the Mises stress was deemed suitable and adopted in all the following discussions.

The folding procedure was simulated in a quasi-static manner using commercial FEA software package Abaqus/Explicit [18]. The sheet was modelled as a 3D deformable shell and meshed with quadrilateral shell elements S4R, whereas the rigid bodies were modelled as 3D analytical rigid parts. The material was chosen to be silicone rubber with linear elastic material properties as follows: density $\rho=930 \mathrm{~kg} / \mathrm{m}^{3}$, Young's modulus $E=7.8 \mathrm{MPa}$, and Poisson's Ratio $v=0.47$ [19].

Convergence tests with respect to mesh density and analysis time, respectively, were also conducted prior to the analysis and checked against two principles recommended by ABAQUS [18]: first of all, the ratio of artificial energy to internal energy was below 5\% to make sure that hour-glassing effect would not significantly affect the results; and secondly, the ratio of kinetic energy to internal energy was below 5\% during most of the folding process to ensure that dynamic effect could be considered 
as insignificant. It was found that an element size of $1 \mathrm{~mm}$ and an analysis time of $0.1 \mathrm{~s}$ were suitable and adopted in the analysis.

\section{Deformation and Stress Analyses}

\subsection{Deformation Analysis}

A series of sheets with $t=0.5 \mathrm{~mm}$ and $d$ varying between $15 \mathrm{~mm}$ to $37.5 \mathrm{~mm}$ are investigated here. It can be seen in Fig. 2(a) that after step 1, path BB' remains straight, while path AA' is deformed into regions: a straight region composed of two overlapped straight parts and a waterdrop-shaped region. Zoomed-in views of the waterdrop-shaped regions of the sheets are plotted in Fig. 2(b), and the largest curvature at the center of the waterdrop-shaped region is measured and plotted against $d$ in Fig. 2(c). The curvature is found to be decreased as $d$ increases, and the relationship between them is approximately linear with a high coefficient of determination $R^{2}=0.9754$.

In step 2 folding, both folding paths deform. Taking the sheet with $d=30 \mathrm{~mm}$ as an instance, when the sheet is folded about path AA', the waterdrop-shaped region is flattened and bent into a U-shape as illustrated in Fig. 3(a), and a local vertex is formed around the intersection point. The deformed profiles of path BB' are shown in Fig. 3(b), and the largest curvature at the center of the bent region is drawn with respect to $d$ in Fig. 3(c). Again, a linear-like relationship exists between the two parameters with a coefficient of determination $R^{2}=0.9267$.

In addition, we have also found that the final shape of a sheet after step 2 folding depends on its thickness. This phenomenon is demonstrated through analyzing a number of sheets with $d$ fixed to $30 \mathrm{~mm}$ but $t$ varying between $0.1 \mathrm{~mm}$ and $1 \mathrm{~mm}$ at an interval of $0.1 \mathrm{~mm}$. Three types of shapes are observed as illustrated in Fig. 4 (a-c). When $t \leq 0.3 \mathrm{~mm}$, a sheet cannot be folded compactly as a triangular surface is formed. The deformed shape of a sheet with $t=0.3 \mathrm{~mm}$ is shown in Fig. 4(a) 
as an instance. The triangular surface disappears when $t$ surpasses $0.3 \mathrm{~mm}$. Then two kinds of vertices, a sharp point-like vertex and a smooth arc-like vertex, could be formed, which are respectively shown in Fig. 4(b) in the case of $t=0.4 \mathrm{mmand} 4(\mathrm{c})$ in the case of $t=0.6 \mathrm{~mm}$. Numerical results indicate that when $0.3 \mathrm{~mm}<t<0.5 \mathrm{~mm}$, a point-like vertex will occur, and when $t \geq 0.5 \mathrm{~mm}$, an arc-like vertex will appear.

The non-compact mode in Fig. 4(a) is undesirable for the purpose of packaging and should therefore be avoided in practice. A partially folded configuration of the non-compact sheet in Fig. 4(a) is drawn in Fig. 4(d). It can be seen that two creases in yellow occur as opposed to the desired crease location in blue, which lead to the triangular surface. A qualitative explanation is that the formation of creases is driven by the distal rigid bodies $1,4,7,10$ and $3,6,9,12$. When a sheet is very thin and soft, it is not able to pass on enough bending moments to the desired crease location, thereby leading to the two yellow creases. Correspondingly, a simple solution is to reduce $d$ so that the desired crease is shorter and easier to form. As also shown in Fig. 4(d), the triangular surface reduces with $d$. When $d$ is decreased to $28.4 \mathrm{~mm}$, the desired crease is formed and a compact folding is obtained. If a thinner sheet is to be compactly folded, a smaller value of $d$ is required to eliminate triangular surfaces. Numerical results show that the maximum value of $d$ to ensure compact folding is $23.4 \mathrm{~mm}$ when $t=0.2 \mathrm{~mm}$, and $14.3 \mathrm{~mm}$ when $t=0.1 \mathrm{~mm}$.

\subsection{Stress Distribution}

A sheet with $t=0.5 \mathrm{~mm}$ and $d=30 \mathrm{~mm}$ is first studied as an instance. The Von Mises stress contour on the undeformed shape of the sheet after step 1 folding is presented in Fig. 5(a). To facilitate analysis, six horizontal paths (denoted by $\mathrm{A} n \mathrm{~A} n$ ') parallel to the $\mathrm{X}$-axis and six vertical ones (denoted by $\mathrm{B} n \mathrm{~B} n$ ') parallel to the Y-axis are defined on the undeformed shape of the sheet, the distance between adjacent paths being selected as $15 \mathrm{~mm}$. The stresses along those paths are 
extracted and drawn in Fig. 5(b) and (c). As expected, a symmetric stress distribution about path BB' is observed, and the stress in the middle area where the folding crease is formed is generally higher than those on the left and right hand sides. Moreover, for the paths crossing rigid bodies, e.g., AA', A3A3', B2B2', B3B3', a significantly larger stress occurs on the spots of the rigid bodies. This is understandable as the rigid bodies control the folding motion and therefore unavoidably incur stress concentration.

When the sheet is cross folded, the Von Mises stress contour on the undeformed shape of the sheet is depicted in Fig. 6(a). Notice that since the stress distributions around the intersection point is of primary interest, only the stresses along the horizontal and vertical paths in the central $40 \times 40 \mathrm{~mm}$ area are plotted in Fig. 6 (b) and (c), respectively. Generally, the symmetry in stress distribution that is observed after step 1 folding is still preserved. In addition, a high stress area is formed in the neighborhood of the intersection point $\mathrm{O}$. The node with the peak stress, $\sigma_{\max }=2.276 \mathrm{MPa}$, is highlighted by the red dot in the zoomed area in Fig.6(a). Notice that this node does not coincide with the intersection point $\mathrm{O}$, but stays very close to it. Considering that the tensile strength of silicon rubber is between $2.4 \mathrm{MPa}$ and $5.5 \mathrm{MPa}$ [19], the peak stress of the sheet is close to the lower bound and hence has potential to cause permanent material deformation.

\subsection{Effects of Geometric Parameters on Peak Stress}

The above analysis has demonstrated that a peak stress will occur in the neighborhood of the intersection point of the two folding creases after step 2 folding. And this peak stress is of concern since it is most likely to cause material plastic deformation. To investigate the effects of geometrical parameters on peak stress, a series of sheets are built and analyzed, with $t$ varying between $0.3 \mathrm{~mm}$ and $0.9 \mathrm{~mm}$ at an interval of $0.1 \mathrm{~mm}$ and $d$ varying from $20 \mathrm{~mm}$ to $30 \mathrm{~mm}$ at an interval of $2.5 \mathrm{~mm}$. The $\sigma_{\max }$ of all the sheets are extracted and drawn in Fig. 7. It can be seen that when $t \leq 0.6 \mathrm{~mm}$, 
the $\sigma_{\max }$ surface is relatively smooth. And two trends hold in general: first of all, $\sigma_{\max }$ increases with $t$, which is understandable as the maximum stress in bending increases with material thickness; and secondly, when $t$ is fixed, the effect of $d$ on $\sigma_{\max }$ is rather weak, suggesting that only the geometry of the sheet plays a major role in determining $\sigma_{\max }$. When $t>0.6 \mathrm{~mm}$, the surface shows a clear sign of disturbance, which is possibly due to the random location of $\sigma_{\max }$ occurring in the sheet. A more detailed analysis is required to fully understand the peak stress in this range.

\section{Peak Stress Relief Methods}

It has shown in the previous section that a cross folded sheet has severe stress concentration and may therefore lead to permanent material deformation. In this section, two methods are proposed and compared to find out an effective way of reducing peak stress in this area.

\subsection{Central Hole Method}

A straightforward method to reduce peak stress is to make the central area of a sheet hollow by cutting a circular hole, whose center is coincident with the sheet's center O, see Fig. 8(a). To test this method, three sheets with identical $d=30 \mathrm{~mm}$ and $t=0.5 \mathrm{~mm}$, but varying hole diameters $d_{0}$ of $1 \mathrm{~mm}, 2 \mathrm{~mm}$, and $4 \mathrm{~mm}$, are built and analyzed. The Von Mises stress contours of the sheets without a hole and with a hole of $d_{0}=1 \mathrm{~mm}$ are drawn in Fig. 8(b) as instances. And the stress distributions along path AA' and BB' in the four cases are respectively plotted in Fig. 8(c) and (d). As expected, the nodes on the edge of a hole experience a significant decrease in stress. However, a new peak stress appears in the neighborhood. Moreover, the $\sigma_{\max }$ in a sheet with a hole could exceed that in the corresponding sheet without a hole when the hole diameter is small, see the case of $d_{0}=1 \mathrm{~mm}$ in Fig. 8(c) and (d). The $\sigma_{\text {max }}$ drops when $d_{0}$ further increases, with a reduction of $9.4 \%$ being achieved in the case of $d_{0}=4 \mathrm{~mm}$. Therefore, the hole in a sheet cannot be too small for the purpose 
of lowering peak stress. On the other hand, the hole cannot be too big to prevent that too much material is removed from the sheet. So there is a trade-off between the introduced peak stress and the size of the hole.

To investigate the influences of geometrical parameters on the peak stress relief effect, a number of sheets with an identical hole of $d_{0}=4 \mathrm{~mm}$, but $t$ varying between $0.3 \mathrm{~mm}$ and $0.9 \mathrm{~mm}$ at an interval of $0.1 \mathrm{~mm}$ and $d$ varying from $20 \mathrm{~mm}$ to $30 \mathrm{~mm}$ at an interval of $2.5 \mathrm{~mm}$, are analyzed, and their peak stresses are plotted in Fig. 9 together with the corresponding intact sheets without a hole. Different from the result for the intact sheets, the sheets with a hole form a relatively smooth $\sigma_{\max }$ surface during the entire range. This result indicates that the introduction of geometric discontinuity in a sheet helps to reduce the degree of randomness and leads to a more predictable response, which is preferable from the design point of view. The two trends observed in the case of intact sheets, i.e., $\sigma_{\max }$ increases with $t$ and only very weakly depends on $d$, are still preserved. This is also a desirable feature as $\sigma_{\max }$ can be estimated purely based on sheet geometry.

Regarding peak stress reduction, it can be seen that the $\sigma_{\max }$ is generally lower than those of the corresponding intact sheets, but the percentage of reduction varies significantly from $2.5 \%$ to $30.1 \%$. This is mainly caused by the disturbance of the $\sigma_{\max }$ surface of the intact sheets.

\subsection{Central Slit Method}

Similar to the central hole method, the central slit method also involves making a cut at the central area of a sheet, but with a different shape. A slit cut composed of a slim rectangle and one small circle at each end is illustrated in Fig. 10(a). The small circles are present to avoid formation of very sharp corners at the ends. 
Two kinds of silts, a horizontal one along path AA' and a vertical one along path BB', are considered. Two sheets with identical $d=30 \mathrm{~mm}$ and $t=0.5 \mathrm{~mm}$, but different slit orientations are analyzed and compared with the corresponding intact one without a slit. The dimension of the slit are as follows: slit length $l_{0}=4 \mathrm{~mm}$ and hole diameter $d_{1}=2 \mathrm{~mm}$. The Von Mises stress contours of the two models are presented in Fig. 10(b), and the stress distributions of them along AA' and BB' are respectively drawn in Fig. 10(c) and (d) along with the corresponding intact sheet. The results show that the effects caused by the two kinds of slits are quite different. The horizontal slit decreases the stress in the area close to it. However, it leads to a significant increase in stress at one end of the slit as shown in Fig. 10(c), resulting in an increase in $\sigma_{\max }$ by $26.3 \%$. The vertical slit, in contrast, reduces the $\sigma_{\max }$ by $33.6 \%$ as shown in both Fig. 10(c) and (d). Therefore, a vertical slit is deemed more effective than a horizontal one.

From the design point of view, it is beneficial to minimize hole diameter and vertical slit length. A number of sheets, which had identical $d=30 \mathrm{~mm}$ and $t=0.5 \mathrm{~mm}$, but varying $d_{1}$ between $0-2 \mathrm{~mm}$ and $l_{0}$ between 4-8mm, were analyzed, and the peak stresses are drawn in Fig. 10(e). As expected, a slit without holes, i.e., $d_{1}=0$, actually leads to an increase in $\sigma_{\max }$. Moreover, the holes need to reach a certain size, $2 \mathrm{~mm}$ in the study here, in order to achieve a substantial peak stress relief. Regarding slit length, the peak stress is found to be insensitive to slit length within the range studied here. And therefore no further increase in slit length beyond $4 \mathrm{~mm}$ is desired.

The influences of geometrical parameters on the peak stress reduction effect by a vertical slit are also investigated through a series of sheet with an identical vertical slit of $l_{0}=4 \mathrm{~mm}$ and $d_{1}=2 \mathrm{~mm}$, but varying $t$ between $0.3 \mathrm{~mm}$ and $0.9 \mathrm{~mm}$ at an interval of $0.1 \mathrm{~mm}$ and $d$ between $20 \mathrm{~mm}$ and $30 \mathrm{~mm}$ at an interval of $2.5 \mathrm{~mm}$. Their peak stresses are extracted and plotted in Fig. 11 together with those 
of the corresponding intact sheets. Similar to the result of the central hole method, the $\sigma_{\max }$ surface also becomes relatively smooth, again confirming the effect of geometries discontinuity in a sheet. Moreover, a $\sigma_{\max }$ reduction of $5.7 \%-34.2 \%$ is achieved. Compared with the central hole method, the central slit method has a smaller hole diameter when a similar $\sigma_{\max }$ reduction is achieved, which might be preferable for design purpose as less material needs to be removed. In the study here, a 50\% less material removal is achieved by the vertical slit.

\section{Conclusion}

In this paper, the cross folding of a flat elastic sheet was numerically studied to investigate the deformation and stress of the sheet. It is found that a vertex forms and brings singularity to the system, which leads to local stress concentration. The peak stress can be reduced by decreasing shell thickness. However, it is shown that the thickness cannot be too small as the sheet may not be folded up compactly as it should be. Two peak stress relief methods, the central hole method and the central slit method, are proposed and analyzed, and a central slit which is oriented parallel to the folding crease that is folded first is found to be most effective with minimal material removal.

The sheets in the study are created with a certain material. The work can be extended to sheets with various materials. In addition, the folding of more practical curved surfaces will also be pursued in the future.

\section{Acknowledgement}

YC would like to thank the financial support from the Natural Science Foundation of China (Projects No. 51422506 and No. 51290293) and Ministry of Science and Technology of China (Project No. 2014DFA70710). JM would like to acknowledge the financial support from the Natural 
Science Foundation of China (Project No. 51575377). Z You wishes to acknowledge the support of Air Force Office of Scientific Research (FA9550-16-1-0339). He was a visiting professor at Tianjin University while this research was carried out.

\section{References}

[1] V. Fraux, M. Lawton, J.R. Reveles, Z. You, Novel large deployable antenna backing structure concepts for foldable reflectors, Ceas Space J. 5 (2013) 195-201.

[2] H. Shi, B. Yang, Nonlinear Deployable Mesh Reflectors, N.A. Approach. Eng. Appl. (2012) 237-263.

[3] A. Meguro, K. Shintate, M. Usui, A. Tsujihata, In-orbit deployment characteristics of large deployable antenna reflector onboard Engineering Test Satellite VIII, Acta. Astronaut. 65 (2009) $1306-1316$.

[4] A. meguro, H. Lshikawa, A. Tsujihata, Study on ground verification for large deployable modular structures, J. Spacecraft Rockets, 43 (2006) 780-787.

[5] M. Jin, Comparative analysis of deployable truss structures for mesh antenna reflectors, Aiaa. J. 36 (2015) 1546-1548.

[6] S. Pellegrino, Deployable membrane reflectors, Nature, 11 (2002) 73-80.

[7] W.D. Reynolds, S.K. Jeon, J.A. Banik, T.W. Murphey, Advanced folding approaches for deployable spacecraft payloads, in: Proceeding of the ASME 2013 International Design Engineering Technical Conferences and Computers and Information in Engineering Conference. American Society of Mechanical Engineers, Portland, Oregon, USA, 2013.

[8] M.C. Natori, N. Katsumata, H. Yamakawa, H. Sakamoto, N. Kishimoto, Conceptual model study using origami for membrane space structures, in: Proceeding of the ASME 2013 International 
Design Engineering Technical Conferences and Computers and Information in Engineering Conference. American Society of Mechanical Engineers, Portland, Oregon, USA, 2013.

[9] B.J. Edmondson, R.J. Lang, S.P. Magleby, L.L. Howell, An offset panel technique for thick rigidily foldable origami, in: Proceeding of the ASME 2014 International Design Engineering Technical Conferences and Computers and Information in Engineering Conference. American Society of Mechanical Engineers, Buffalo, New York, USA, 2014.

[10] L. Wilson, S. Pellegrino, R. Danner, Origami sunshield concepts for space telescopes, in: Proceedings of the 54th AIAA/ASME/ASCE/AHS/ASC Structures, Structural Dynamics, and Materials Conference, Boston, Massachusetts, USA, 2013.

[11] M. Thomson, AstroMesh ${ }^{\mathrm{TM}}$ deployable reflectors for ku and ka band commercial satellites, in: Proceedings of the 20th AIAA international communication satellite systems conference and exhibit, Montreal, Quebec, Canada, 2002.

[12] W.J. Rits, Self-supporting shell for use in space, U.S. Patent No. 5,515,067. 7 May 1996.

[13] K. Miura, Method of Packaging and Deployment of Large Membranes in Space, The Institute of Space and Astronautical Science report 618 (1985) 1-9.

[14] M. Schenk, S.D. Guest, Geometry of Miura-folded metamaterials, in: Proceedings of the National Academy of Sciences of the United States of America 110(9) (2013) 3276.

[15] J. Ma, D. Hou, Y. Chen, Z. You, Quasi-static axial crushing of thin-walled tubes with a kite-shape rigid origami pattern: Numerical simulation, Thin-Walled Structures 100 (2016) 38-47.

[16] A. Rao, S. Tawfick, M. Shlian, A.J. Hart, Fold Mechanics of Natural and Synthetic Origami Papers, in: Proceeding of the ASME 2013 International Design Engineering Technical Conferences 
and Computers and Information in Engineering Conference. American Society of Mechanical Engineers, Portland, Oregon, USA, 2013.

[17] H. Zheng, D.F. Liu, C.F. Lee, L.G. Tham, Displacement - controlled method and its applications to material non - linearity, Int. J. Numer. Anal. Methods. Geomech. 29 (2005) 209-226. [18] Abaqus Analysis User's Manual, Abaqus Documentation Version 6.14-3, 2016, Dassault Systems SIMULIA Corp., Providence, RI, USA.

[19] Granta Design Limited. (2016). Silicone Rubber. Retrieved from AZoM.com: http://www.azom.com/properties.aspx?ArticleID $=920$ 


\section{Captions of Figures}

Fig. 1 Illustration of folding procedure.

Fig. 2 (a) The deformed sheet with $d=30 \mathrm{~mm}$ and the profiles of $\mathrm{AA}^{\prime}$ at varying $d$, (b) the waterdrop-shaped regions of $\mathrm{AA}^{\prime}$ (Zoomed-in) at varying $d$, and (c) the curvature of the waterdrop-shaped region of AA' vs. $d$ after step 1 folding.

Fig. 3 (a) The deformed sheet with $d=30 \mathrm{~mm}$, (b) the profiles of BB' at varying $d$, and (c) the curvature of the bent region of BB' vs. $d$ after step 2 folding.

Fig. 4 Cross folded sheets with identical $d=30 \mathrm{~mm}$, but different thicknesses (a) $t=0.3 \mathrm{~mm}$, (b) $t=0.4 \mathrm{~mm}$, (c) $t=0.6 \mathrm{~mm}$, and (d) partially folded configurations of sheets with identical $t=0.3 \mathrm{~mm}$, but varying $d$.

Fig. 5 (a) The Von Mises stress contour on the undeformed sheet, (b) stress distributions along horizontal paths, and (c) stress distributions along vertical paths after step 1 folding.

Fig. 6 (a) Von Mises stress contour on the undeformed sheet, (b) stress distributions along horizontal paths, and (c) stress distributions along vertical paths after step 2 folding.

Fig. 7 The peak stresses of sheets with $t$ varying between $0.3 \mathrm{~mm}$ and $0.9 \mathrm{~mm}$, and $d$ varying between $20 \mathrm{~mm}$ and $30 \mathrm{~mm}$

Fig. 8 (a) Illustration of the central hole method, (b) Von Mises stress contours of sheets without a hole and with a hole of $d_{0}=1 \mathrm{~mm}$, respectively, (c) stress distributions along AA', and (d) stress distributions along BB' of sheets without a hole and with a hole of $d_{0}=1,2$, $4 \mathrm{~mm}$, respectively, after step 2 folding.

Fig.9 Comparison of the peak stresses of sheets with a hole of $d_{0}=4 \mathrm{~mm}$ and sheets without a hole. 
Fig.10 (a) Illustration of the central slit method, (b) Von Mises stress contours of sheets with a horizontal slit and with a vertical slit, respectively, (c) stress distributions along AA', (d) stress distributions along $\mathrm{BB}^{\prime}$ of sheets without a slit, with a horizontal slit, and with a vertical slit, respectively, after step 2 folding, and (e) Peak stresses of sheets with a vertical slit and varying $d_{0}$ and $l_{0}$.

Fig.11 Comparison of the peak stresses of sheets with a vertical slit and sheets without a slit. 


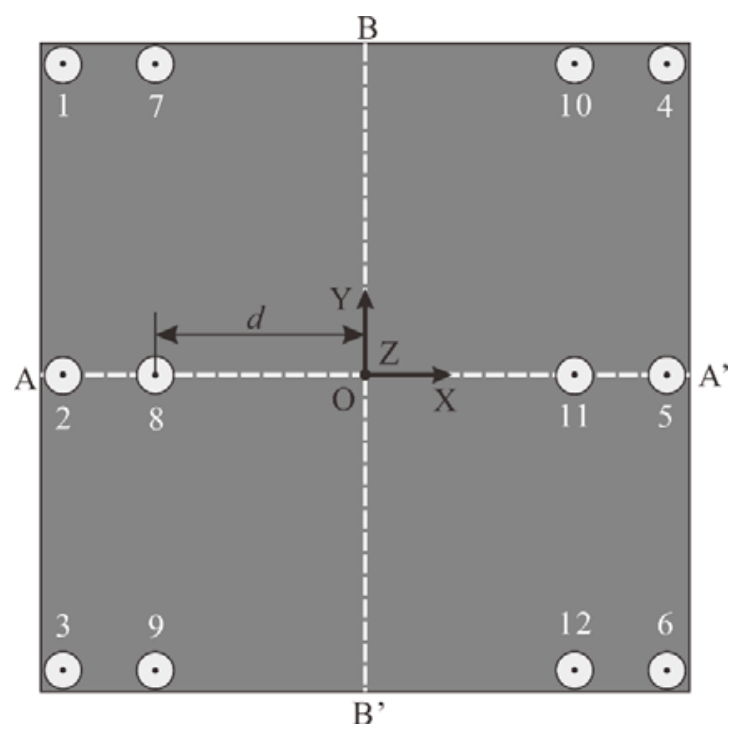

Fig. 1 Illustration of folding procedure. 

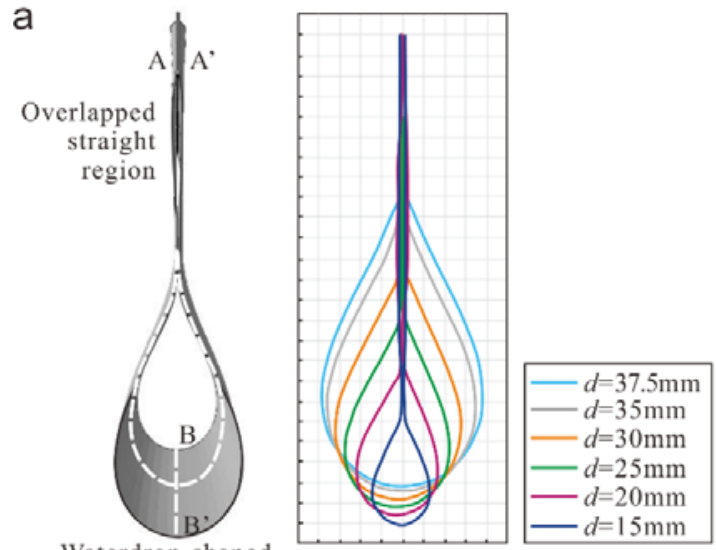

$$
\text { region }
$$

b

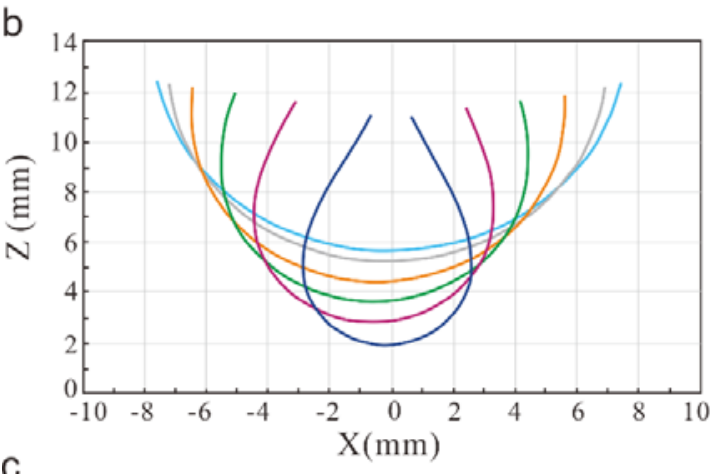

C

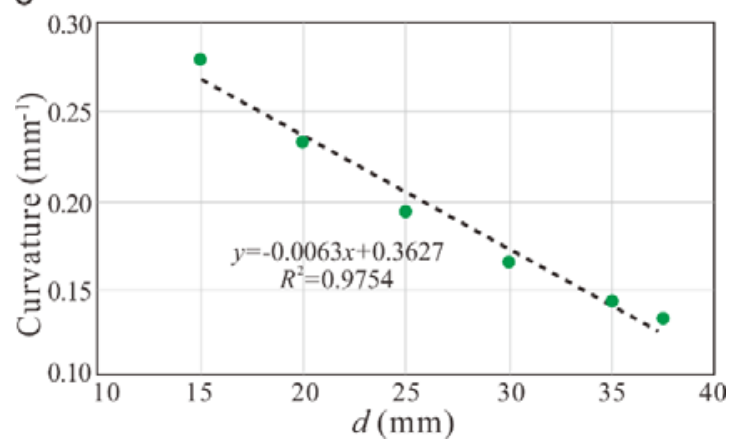

Fig. 2 (a) The deformed sheet with $d=30 \mathrm{~mm}$ and the profiles of AA' at varying $d$, (b) the waterdrop-shaped regions of AA' (Zoomed-in) at varying $d$, and (c) the curvature of the waterdrop-shaped region of AA' vs. $d$ after step 1 folding. 
a

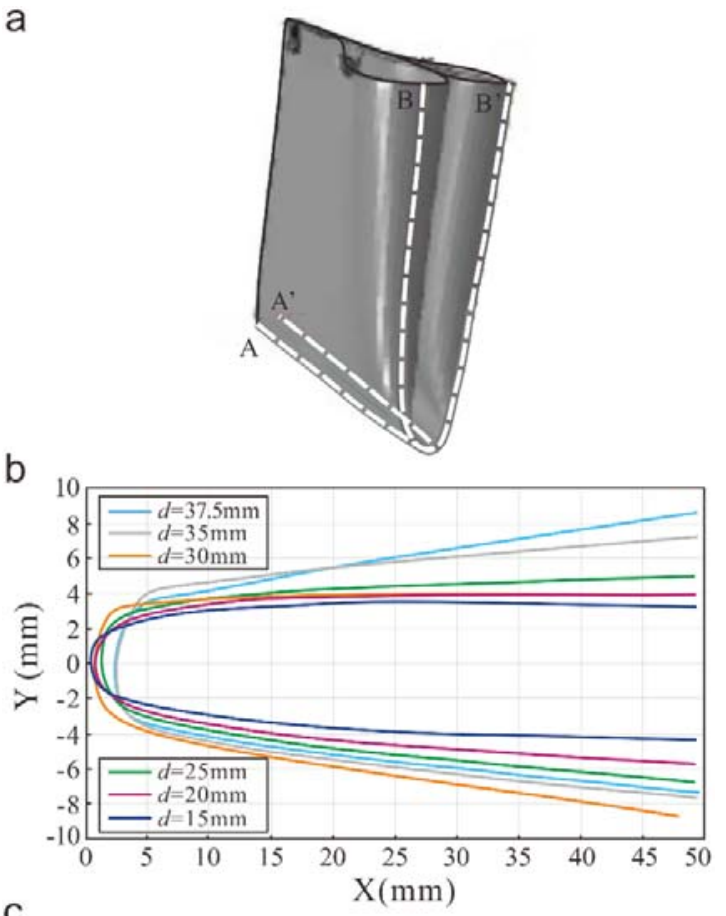

C

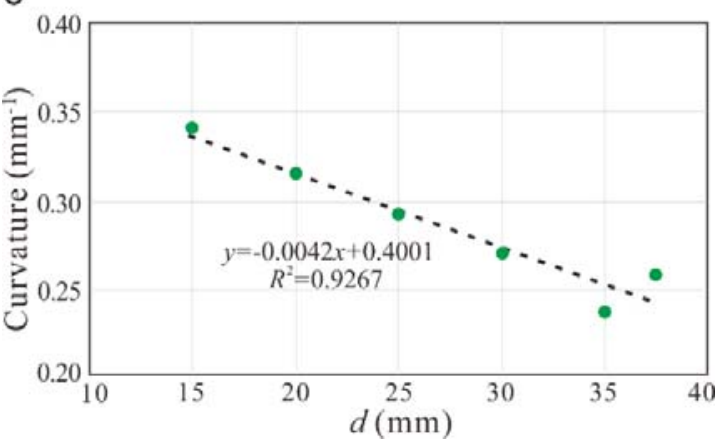

Fig. 3 (a) The deformed sheet with $d=30 \mathrm{~mm}$, (b) the profiles of BB' at varying $d$, and (c) the curvature of the bent region of $\mathrm{BB}^{\prime}$ vs. $d$ after step 2 folding. 
a

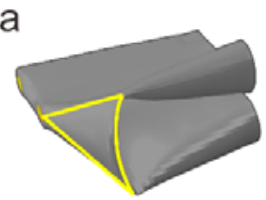

d

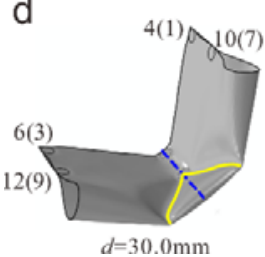

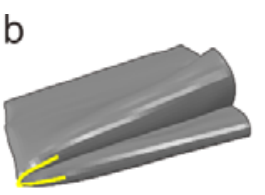

C
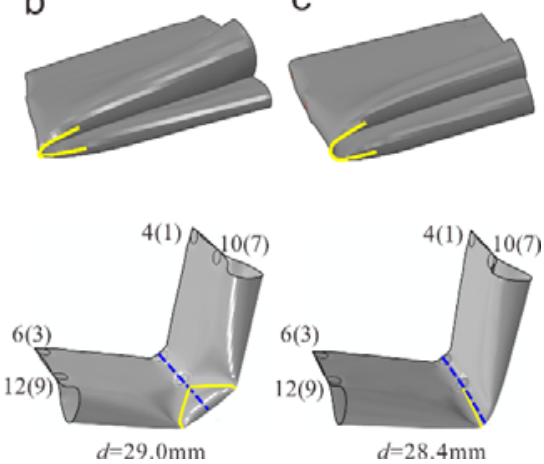

Fig. 4 Cross folded sheets with identical $d=30 \mathrm{~mm}$, but different thicknesses (a) $t=0.3 \mathrm{~mm}$, (b) $t=0.4 \mathrm{~mm}$, (c) $t=0.6 \mathrm{~mm}$, and (d) partially folded configurations of sheets with identical $t=0.3 \mathrm{~mm}$, but varying $d$. 


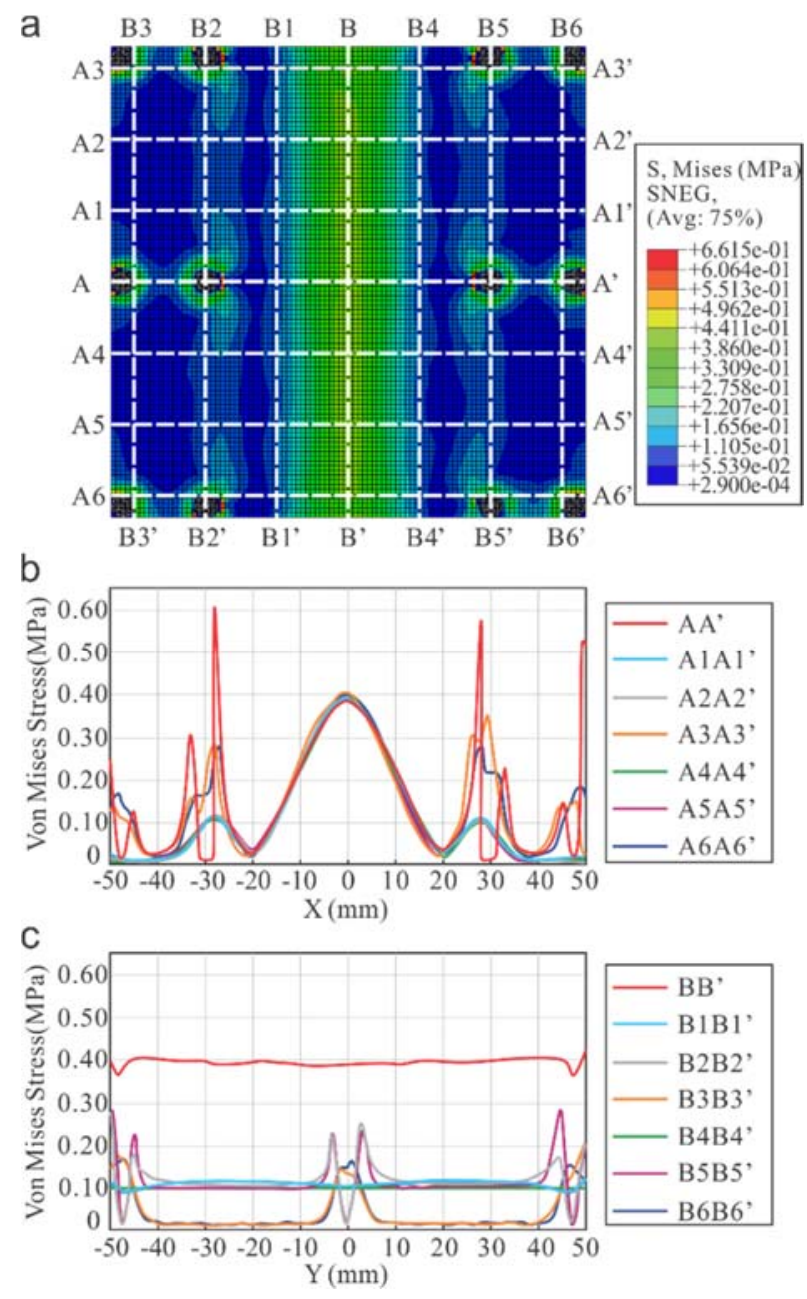

Fig. 5 (a) Von Mises stress contour on the undeformed sheet, (b) stress distributions along horizontal paths, and (c) stress distributions along vertical paths after step 1 folding. 

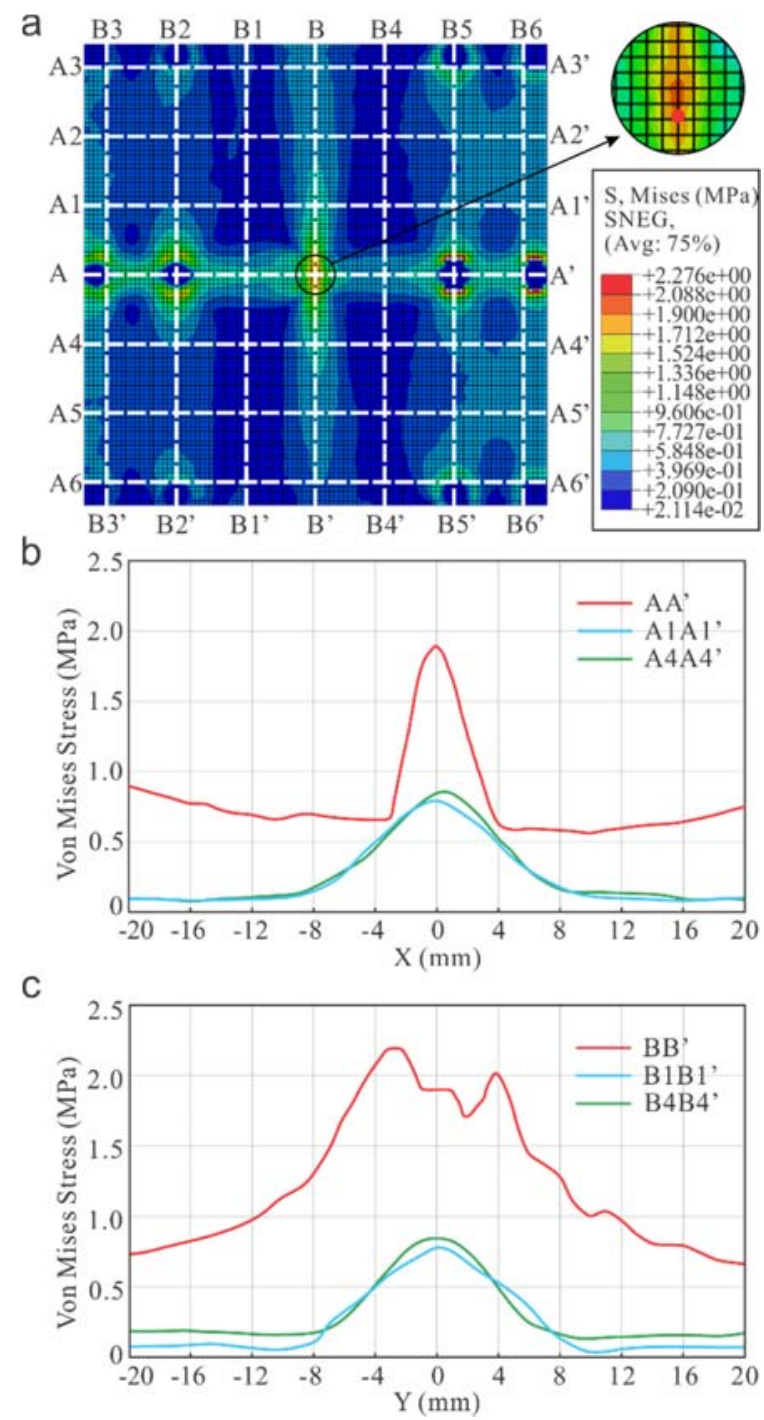

Fig. 6 (a) Von Mises stress contour on the undeformed sheet, (b) stress distributions along horizontal paths, and (c) stress distributions along vertical paths after step 2 folding. 


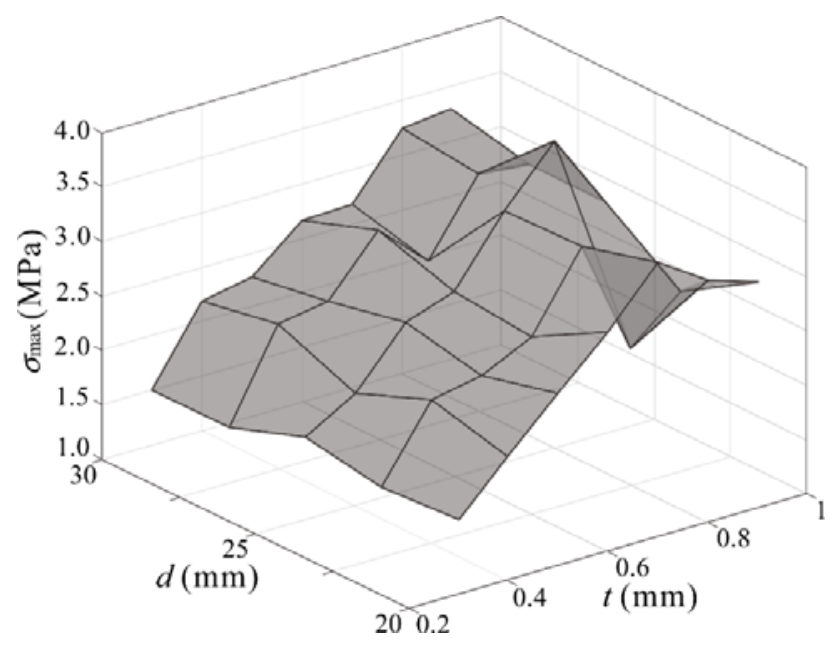

Fig. 7 The peak stresses of sheets with $t$ varying between $0.3 \mathrm{~mm}$ and $0.9 \mathrm{~mm}$, and $d$ varying between $20 \mathrm{~mm}$ and $30 \mathrm{~mm}$. 

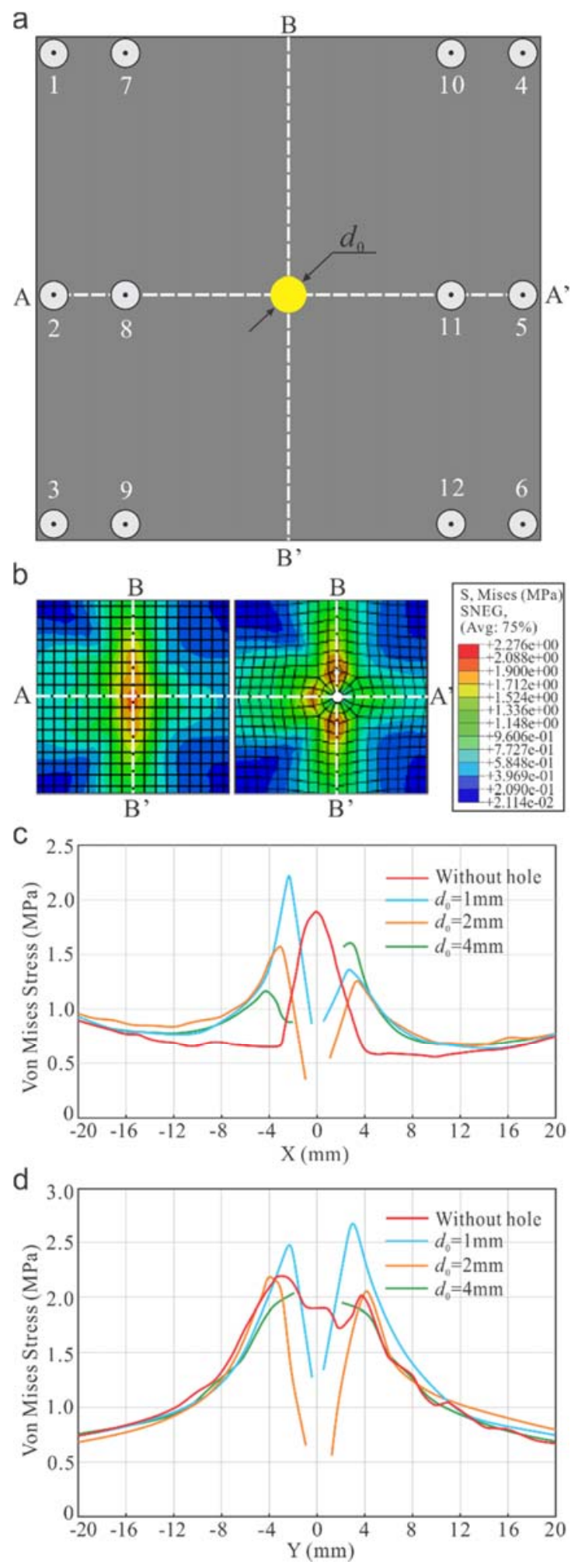

Fig. 8 (a) Illustration of the central hole method, (b) Von Mises stress contours of sheets without a hole and with a hole of $d_{0}=1 \mathrm{~mm}$, respectively, (c) stress distributions along AA', and (d) stress distributions along BB' of sheets without a hole and with a hole of $d_{0}=1,2,4 \mathrm{~mm}$, respectively, after step 2 folding. 


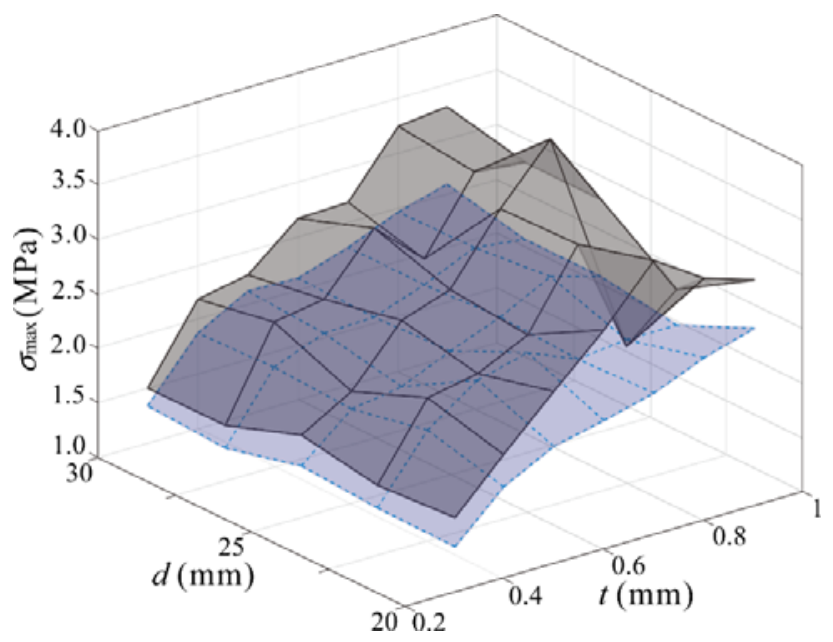

Fig.9 Comparison of the peak stresses of sheets with a hole of $d_{0}=4 \mathrm{~mm}$ and sheets without a hole. 
a

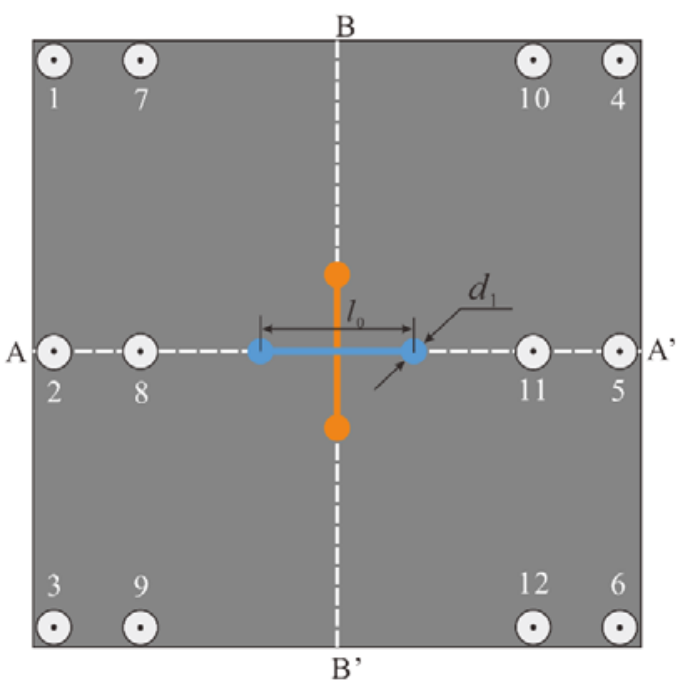

b

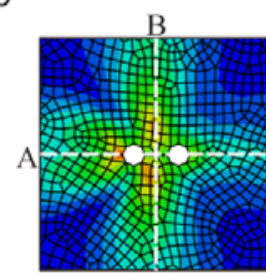

B'

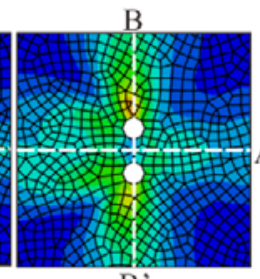

B'

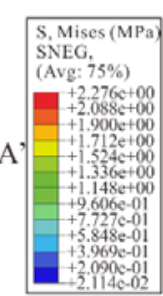

C
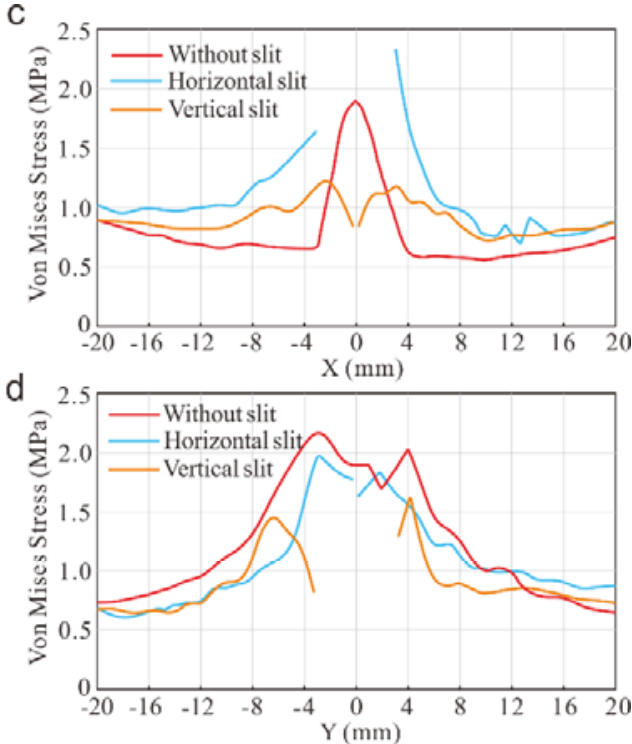

e

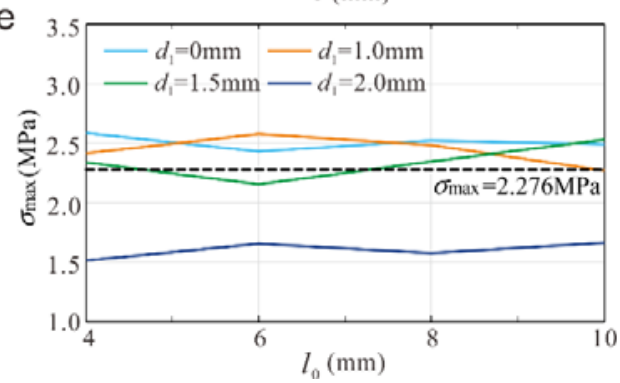

Fig.10 (a) Illustration of the central slit method, (b) Von Mises stress contours of sheets with a horizontal slit and with a vertical slit, respectively, (c) stress distributions along AA', (d) stress distributions along BB' of sheets without a slit, with a horizontal slit, and with a vertical slit, respectively, after step 2 folding, and (e) Peak stresses of sheets with a vertical slit and varying $d_{0}$ and $l_{0}$. 


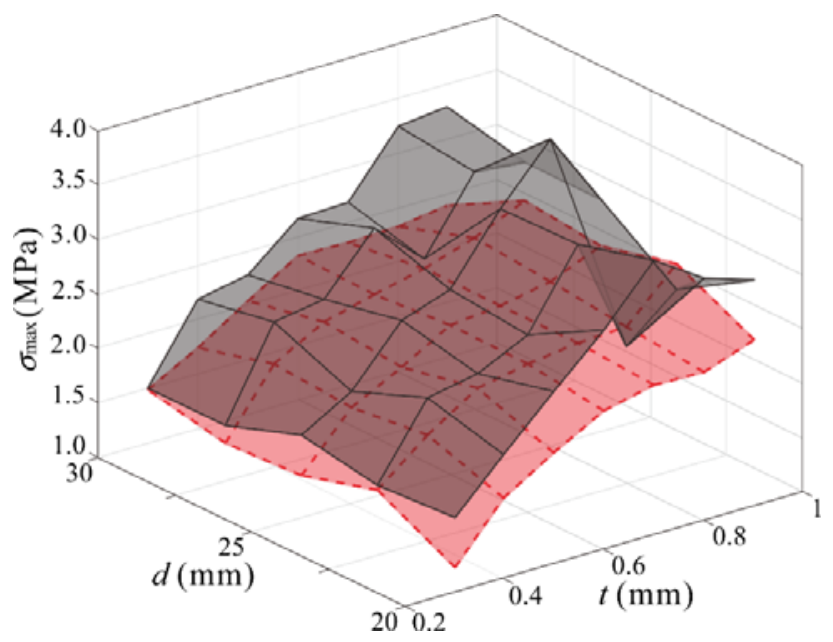

Fig.11 Comparison of the peak stresses of sheets with a vertical slit and sheets without a slit. 